\title{
Electrophoretic karyotypes of clinically isolated yeasts of Candida albicans and $C$. glabrata
}

\author{
Kohtaro Asakura, ${ }^{1}$ Shin-Ichi Iwaguchi, ${ }^{2}$ Michio Homma, ${ }^{2 *}$ Tomoko Sukai, ${ }^{3}$ \\ KOJI HIGASHIDE ${ }^{3}$ and KENJI TANAKA ${ }^{2}$ \\ ${ }^{1}$ Department of Ophthalmology, and ${ }^{2}$ Laboratory of Medical Mycology, Research Institute for Disease Mechanism and \\ Control, Nagoya University School of Medicine, Showa-ku, Nagoya 446, Japan \\ ${ }^{3}$ Department of Obstetrics and Gynaecology, Chita City Hospital, Chita 478, Japan
}

(Received 5 February 1991; revised 25 June 1991; accepted 25 July 1991)

\begin{abstract}
One-hundred-and-four isolates of yeast were collected from the vaginas of 97 outpatients. The isolates were identified by their characteristics in a carbohydrate assimilation test, a serological test and from their morphology. Candida albicans and Candida glabrata were the major isolates (75\% and $20 \%$, respectively). The karyotypes of the isolates were analysed by pulsed-field gel electrophoresis and almost all the karyotypes were distinguishable from one another when the band mobilities were carefully compared. Characteristics and karyotypes were not directly correlated, but seven $\boldsymbol{C}$. albicans isolates (from six patients) had a common atypical karyotype and shared the same phenotype. These isolates are inferred to be generated by a wide genomic reorganization and mutation and the phenotypic changes may be advantageous for survival. The karyotypes of the isolates recovered from individual patients after intervals of 1-6 months were all identical except for one or two highly variable bands which were identified with an rDNA probe. This suggests that the variable bands are too variable to be useful for distinguishing strains, but from the patterns of the identical bands (i.e. except for the variable bands) we concluded that strains from individual patients do not change, at least over short periods. This, coupled with the extensive inter-isolate variability in karyotype, will be useful for Candida source determination and epidemiological studies.
\end{abstract}

\section{Introduction}

Epidemiological studies of Candida infection require the precise identification of individual isolates and clarification of strain delineation (Merz, 1990). The methods used are based on phenotypic biotyping schemes, resistograms, killer types, and mitochondrial and chromosomal DNA restriction endonuclease polymorphisms. These do not permit very specific strain delineation. Recently, a wide variety of DNA polymorphisms has been shown in restriction fragments of repeated DNA sequences (Fox et al., 1989; Scherer \& Stevens, 1987; Soll et al., 1989) and in the chromosomalsized DNAs separated by pulsed-field gel electrophoresis (PFGE) (Kaufmann \& Merz, 1989; Mahrous et al., 1990; Merz et al., 1988; Monod et al., 1990). These polymorphisms have made it possible to distinguish strains efficiently.

C. albicans is one of the organisms in which karyotypes analysed by PFGE have been most studied (Scherer \&

Abbreviation: PFGE, pulsed-field gel electrophoresis.
Magee, 1990). It is considered to be a diploid organism with a haploid number of eight as determined from karyotype analysis and hybridization experiments (Lasker et al., 1989; Magee et al., 1988; Iwaguchi et al., 1990). Recently, we demonstrated that individual DNA probes usually hybridized to one or two chromosome bands and could be used to identify homologous chromosomes with variable positions in electrophoretic karyotypes (Iwaguchi et al., 1990). With the exception of the probe MGL1, when two bands were detected by one probe, the size of one of them was highly conserved whilst the size of the other was fairly variable. It is inferred that the karyotype polymorphism among strains derived mainly from wide size heterogeneity in one of the homologous chromosomes. The chromosomes which hybridized to probe MGL1 were much more variable in size and are here called the variable chromosomes. These results from our previous study (Iwaguchi et al., 1990) were obtained by analysing stock strains subcultured in the laboratory for a long period. In order to assess whether karyotype analysis is a practically useful method for strain delineation and clinical application, it is 
necessary to examine the extent of variation among fresh clinical isolates. In this study, therefore, we analysed the electrophoretic karyotypes of yeast strains, mostly $C$. albicans and C. glabrata, that had been freshly isolated from the vaginas of hospital out-patients.

\section{Methods}

Reference strains. Chromosomes of Saccharomyces cerevisiae (X21801 A ; Mortimer \& Schild, 1985) and Schizosaccharomyces pombe $\left(975-\mathrm{h}^{+}\right.$; Vollrath \& Davis, 1987) were used as reference markers for the molecular size of the chromosome bands in PFGE. FC18 (Magee et al., 1988) and TIMM1063 (Collection of Teikyo University Research Centre for Medical Mycology, Japan) were used as the standard strains of $C$. albicans and C. glabrata, respectively.

Media. The media used were Sabouraud glucose agar ( $1 \%$ peptone, $4 \%$ glucose and $1.5 \%$ agar, all $w / v)$, YPD broth ( $1 \%$ peptone, $1 \%$ yeast extract and $2 \%$ glucose, all w/v), YPD agar $(1.5 \% \mathrm{w} / \mathrm{v}$, agar in YPD broth), cornmeal/Tween 80 agar $[1.7 \mathrm{~g}$ of cornmeal agar (Gibco) and $10 \mathrm{ml}$ Tween $\left.80 \mathrm{l}^{-1}\right]$, and YNB/BSA medium [ $1.7 \mathrm{~g}$ yeast nitrogen base without amino acids and ammonium sulphate (Difco), $20 \mathrm{~g}$ glucose and $2 \mathrm{~g} \mathrm{BSA} \mathrm{1}^{-1}$ ].

Isolation of yeasts from the vagina. The 104 isolates of yeast were collected from 97 outpatients of the Department of Obstetrics and Gynaecology, Chita City Hospital, Japan, between November 1988 and June 1989. Samples were obtained with sterile cotton swabs and streaked onto Sabouraud glucose agar plates containing $0.1 \mathrm{mg}$ chloramphenicol ml-1. The plates were incubated at $37^{\circ} \mathrm{C}$ for $2 \mathrm{~d}$. The colonies which appeared were restreaked on YPD agar plates and the strain was given a $\mathrm{TCH}$ number.

Identification of isolated yeasts. Colonies (at least two) of each isolate were observed by microscopy to confirm yeast morphology; their electrophoretic karyotypes were then analysed (see below). Each isolate was tested for assimilation of 19 carbohydrates using a commercial kit, the API 20c aux (API Systems) (Buesching et al., 1979). A seven-digit profile number based on the assimilation reactions and hyphal formation was determined according to the instructions provided by the manufacturer. The number was matched to the profile index of species provided by the manufacturer.

In addition, each isolate was tested for antigenic type using a commercial kit, Candida Check (Iatron Laboratories, Japan) (Shinoda et al., 1981). This system is designed to identify eight medically important species of Candida according to their agglutination reactions with the ten-factor sera.

Extracellular proteinase assay. Isolates were grown in YPD broth at $37^{\circ} \mathrm{C}$. Stationary-phase cultures were inoculated into YNB/BSA medium at $1 / 100$ dilution. After $48 \mathrm{~h}$, the cells were sedimented by centrifugation and $0.1 \mathrm{ml}$ of the supernatant or YNB/BSA medium (as a control) was mixed with $1 \mathrm{ml}$ citrate buffer ( $\mathrm{pH} \mathrm{3.6)}$ containing $1 \%$ $(w / v) ~ B S A$. The mixture was incubated for $40 \mathrm{~min}$ at $37^{\circ} \mathrm{C}$. To stop the reaction, $1 \mathrm{ml}$ of $0.44 \mathrm{M}$-TCA was added. After $10 \mathrm{~min}$ at room temperature, the mixture was centrifuged for $5 \mathrm{~min}$ at 3000 r.p.m. The absorbance of the supernatant at $280 \mathrm{~nm}$ was measured.

Preparation of yeast chromosomal DNA. Yeast chromosomal DNA for PFGE was prepared as described previously (Iwaguchi et al., 1990).

Pulsed-field gel electrophoresis (PFGE). The yeast chromosomal DNA was separated by contour-clamped homogeneous electric field (CHEF) electrophoresis which was carried out with the Pulsaphor system using a hexagonal electrode array (Pharmacia-LKB) or the
CHEF-DRII system (Bio-Rad Laboratories) as described previously (Iwaguchi et al., 1990).

Southern hybridization. Southern hybridization was performed using probe MGLl and an rDNA probe as described previously (Iwaguchi $e t$ al., 1990). The rDNA probe was prepared from plasmid pAT68, containing rDNA of Zygosaccharomyces bailli which strongly hybridizes to $C$. albicans DNA (Sugihara et al., 1986). The MGL1 probe was prepared as described previously (Iwaguchi et al., 1990).

\section{Results}

\section{Characterization of yeasts from the vagina}

Isolated yeasts were identified by a carbohydrate assimilation test, a serological test and a morphology test. Characteristics of the isolates are listed in Table 1.

C. albicans comprised $75 \%$ ( 78 isolates) of the total isolates. Sixty-nine isolates of $C$. albicans had an API index of 2576174 which corresponds to a 'very good' $(99.7 \%)$ identification accuracy. Although strains with the indexes 2144174,6172114 and 2542034 were not correlated with a species according to the manufacturer's index, they were assigned to $C$. albicans on the basis of the results from (i) a serological test (Table 1), (ii) electrophoretic karyotype analysis (see below) and (iii) a hybridization test with the $C$. albicans-specific probes LYS2, BEN1 (= BENr) and MGL1, which have been characterized previously (Iwaguchi et al., 1990). The hybridization signals from these probes were as strong from the isolate DNAs as they were from the DNA of $C$. albicans standard strain FC18 (data not shown).

Table 1. Characterization of yeasts isolated from the vagina

\begin{tabular}{|c|c|c|c|c|}
\hline Species & $\begin{array}{l}\text { No. of } \\
\text { strains }\end{array}$ & $\begin{array}{c}\text { API } \\
\text { index* }\end{array}$ & $\begin{array}{c}\text { Serotype } \\
(\mathrm{A} / \mathrm{B}) \dagger\end{array}$ & $\begin{array}{c}\text { Extracellular } \\
\text { proteinase } \ddagger\end{array}$ \\
\hline C. albicans & 69 & 2576174 & $\mathrm{D}(61 / 8)$ & $+\S$ \\
\hline C. albicans & 1 & 2144174 & D $(1 / 0)$ & + \\
\hline C. albicans & 1 & 6172114 & D $(1 / 0)$ & + \\
\hline C. albicans & 7 & 2542034 & D $(0 / 7)$ & + \\
\hline C. glabrata & 21 & 2000040 & D & - \\
\hline C. krusei & 1 & 6000104 & D & NT \\
\hline C. parapsilosis & 1 & 6756175 & D & NT \\
\hline S. cerevisiae & 1 & 2044032 & ND & NT \\
\hline R. rubra & 1 & 2470072 & ND & NT \\
\hline Cr. uniguttulatus & 1 & 2707173 & ND & NT \\
\hline
\end{tabular}

* Defined by a test based on the assimilation of 19 carbohydrates and on hyphal formation using a commercial kit (API 20c aux).

$\dagger$ The serological test was performed with a commercial kit (Candida Check). D means that the species was defined by this test; ND, Not defined. The number of strains with serotypes $A$ and $B$, which were defined in C. albicans, is shown in parenthesis.

†xtracellular proteinase activity required for growth in YNB/BSA medium was determined and the proteinase was detected on an SDSPAGE gel (not shown). NT, Not tested.

$\S$ One of these 69 strains was proteinase negative. 
Twenty-one isolates were identified as $C$. glabrata. All had the API index 2000040. One isolate had an API index of 6000104 , suggesting it was $C$. krusei or $C$. lipolytica; serological testing supported the former identification. One isolate was confirmed as $C$. parapsilosis, based on the API index and its serological profile. One isolate each of $S$. cerevisiae, Rhodotorula rubra and Cryptococcus uniguttulatus were assigned on the basis of the API index alone.

Extracellular proteinase activity of $C$. albicans and $C$. glabrata isolates was detected by their ability to grow in YNB/BSA broth, which contains BSA as sole nitrogen source. None of the $C$. glabrata isolates grew in this broth. All but one of the C. albicans isolates grew, though proteinase activities of their cultures (data not shown) were variable, and some of them were very low.

\section{Electrophoretic karyotypes of C. albicans}

The electrophoretic karyotypes of the 78 C. albicans isolates were compared with that of a standard strain (FC18) under conditions (Fig $1 a$ ) in which their chromosomes were well separated over the entire size range in a single gel, and also under conditions (Fig. $1 b$ ) in which chromosomes smaller than $2.0 \mathrm{Mb}$ were better separated. The karyotypes of many isolates were similar to that of $\mathrm{FC18}$, whose chromosome sizes have been calibrated as $2 \cdot 86,2.66,2 \cdot 20,1.90,1 \cdot 70,1 \cdot 36,1 \cdot 18$ and $1.08 \mathrm{Mb}$. These chromosomal bands each represent different chromosomal DNA molecules except the largest band $(2.86 \mathrm{Mb})$, which is a mixed band containing both homologues of one chromosome as well as one homologue of the second largest $(2.66 \mathrm{Mb})$ chromosome (Iwaguchi et al., 1990). The sizes and numbers of the chromosome bands resolved under the former conditions (Fig $1 a$ ) ranged from 0.3 to $3.0 \mathrm{Mb}$ and from 6 to 13 bands. There were 6 bands in one isolate, 7 bands in 13 isolates, 8 bands in 25 isolates, 9 bands in 19 isolates, 10 bands in 10 isolates, 11 bands in two isolates, 12 bands in two isolates and 13 bands in six isolates. The isolates with 8 bands, whose molecular sizes ranged from 1.0 to $2.8 \mathrm{Mb}$, were the most frequently detected. The same or similar-sized 7 bands (excluding the $2.66 \mathrm{Mb}$ band), which we call the 7 common bands, were usually present in most isolates. The $2.66 \mathrm{Mb}$ band and $2.86 \mathrm{Mb}$ band of FCl 8 were identified by probe MGL1 as containing homologues of chromosome 2 ; chromosome bands identified by probe MGL1 were very variable in size among stock strains (Iwaguchi et al., 1990) and we call these the variable bands. We found that an rDNA probe hybridized to the same chromosome bands as those which hybridized to probe MGLl (data not shown), and so the variable bands in the isolates
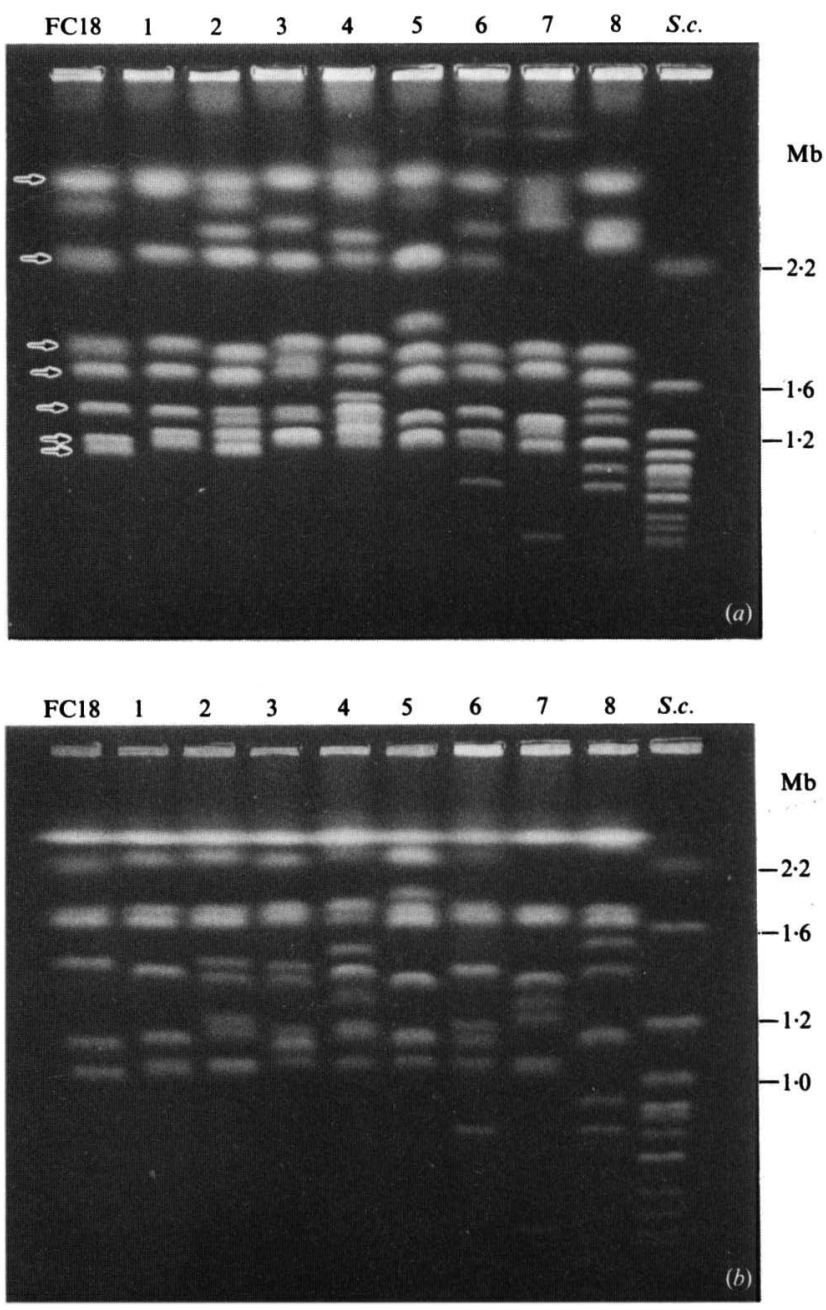

Fig. 1. Separation of chromosomal DNAs of C. albicans. PFGE was run under conditions of switching intervals, voltages and duration times: (a) $300 \mathrm{~s}$ for $24 \mathrm{~h}$ at $140 \mathrm{~V}$, then $1200 \mathrm{~s}$ for $48 \mathrm{~h}$ at $80 \mathrm{~V}$; (b) $100 \mathrm{~s}$ for $15 \mathrm{~h}$ at $180 \mathrm{~V}$, then $300 \mathrm{~s}$ for $20 \mathrm{~h}$ at $140 \mathrm{~V}$. The gels were $0.8 \%$ agarose and were run using the Pulsaphor system (Pharmacia-LKB). The samples were prepared from $C$. albicans strains $\mathrm{FC} 18$, TCH215 (lane 1), TCH231 (lane 2), TCH145 (lane 3), TCH13 (lane 4), TCH7l (lane 5), TCH168 (lane 6), TCH4 (lane 7), TCH219 (lane 8), and from Saccharomyces cerevisiae (S.c.). The API index of TCH168 is 2144174 and the index of the other C.albicans strains is 2576174 . Arrows on the left indicate the 7 common bands of $C$. albicans; pointers on the right indicate chromosomal DNA molecular size markers from $S$. cerevisiae.

described here were characterized using the rDNA probe (Fig. 2, lanes 1-8).

The karyotype variation was rather continuous, but almost all karyotypes of the isolates were distinguishable from each other when the band mobilities were carefully compared under the conditions of Fig. $1(a)$ and Fig. $1(b)$, even without taking into account the variable bands (see Discussion).

The karyotypes of strains with API indexes of 

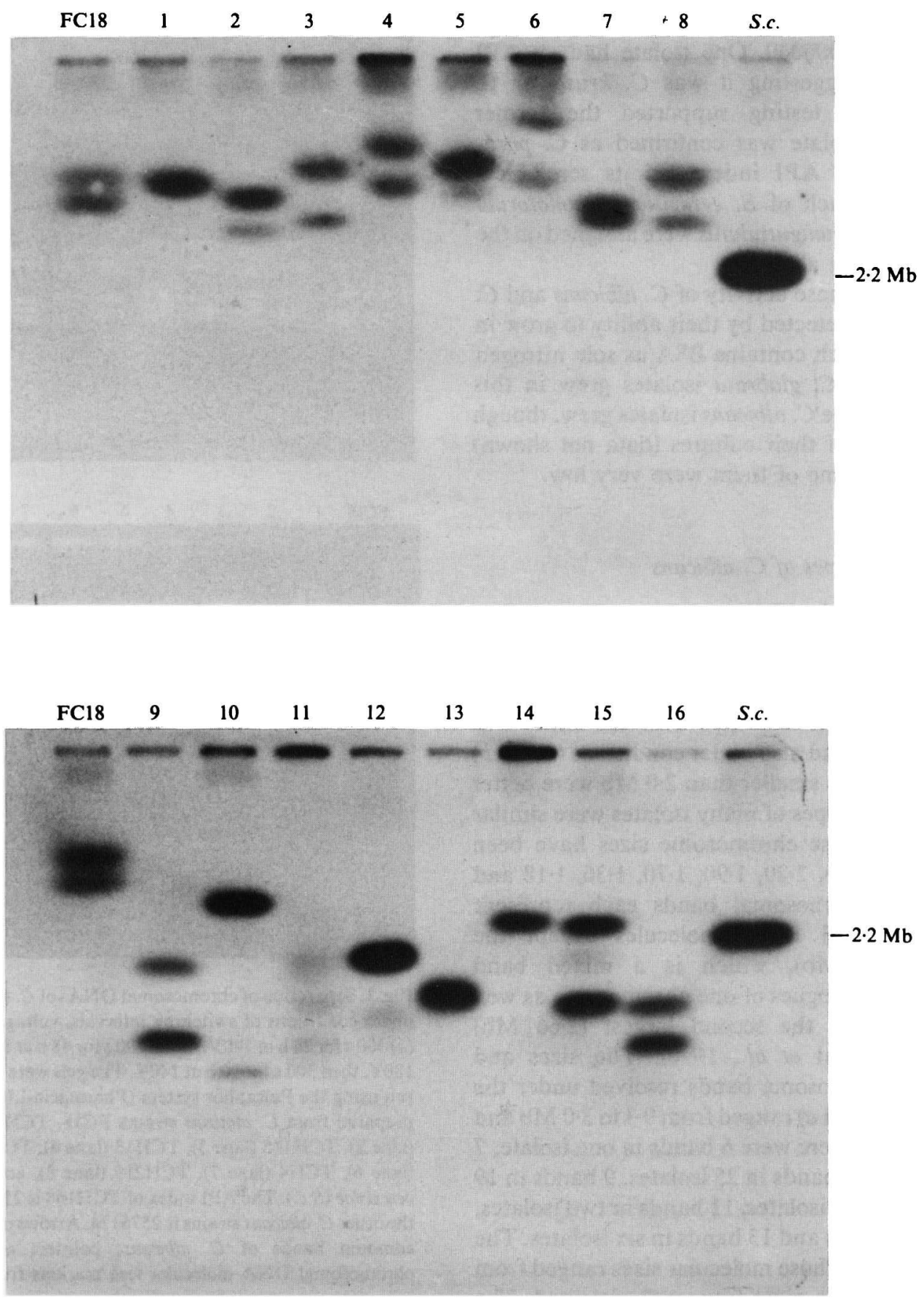

Fig. 2. Assignment of the variable chromosome bands by use of the rDNA probe. The chromosomal DNAs were separated under the conditions used in Fig. $1(a)$ and hybridized by rDNA probe. The samples were prepared from $C$. albicans strains FC18, TCH215 (lane 1), TCH231 (lane 2), TCH145 (lane 3), TCH13 (lane 4), TCH71 (lane 5), TCH168 (lane 6), TCH4 (lane 7), TCH219 (lane 8), from $C$ glabrata strains TIMM1063 (lane 9), TCH1 (lane 10), TCH26 (lane 11), TCH58 (lane 12), TCH157 (lane 13), TCH 198 (lane 14), TCH201 (lane 15), TCH220 (lane 16), and from S. cerevisiae (S.c.). The pointer on the right margin indicates chromosome XII of S. cerevisiae.

2576174,2144174 and 6172114 were all rather similar to that of FC18. On the other hand, the seven isolates (from six patients) with the API index 2542034 had karyotypes that were very different from $\mathrm{FCl} 18$ but were similar to one another (Fig. 3). Their chromosomal DNAs were resolved into 12 or 13 bands. The 7 common bands were present in these isolates together with 5 or 6 additional bands. 


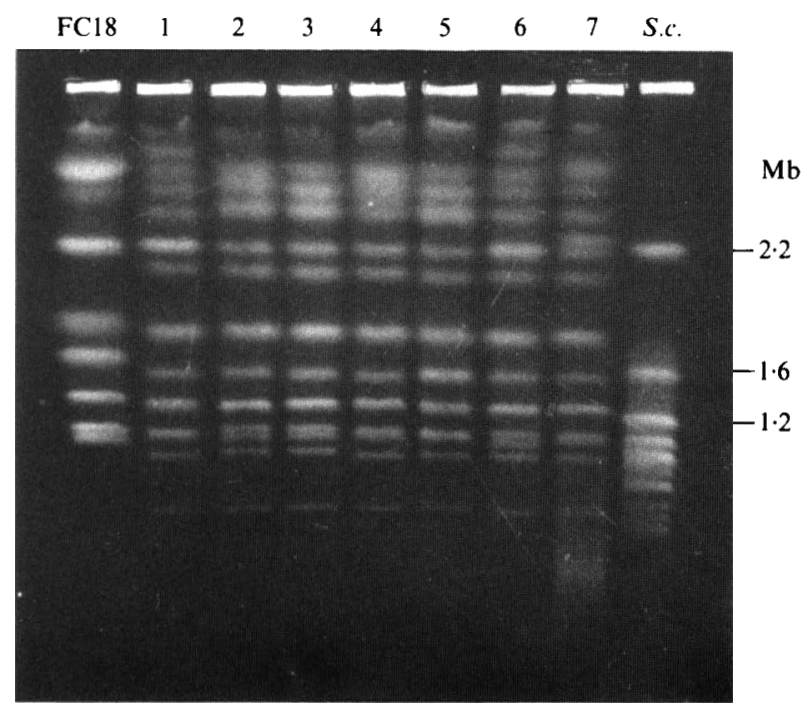

Fig. 3. Atypical karyotypes of $C$. albicans. PFGE was run under the conditions of Fig. 1(a). The sample was prepared from $C$. albicans strains FC18, TCH23 (lane 1), TCH44 (lane 2), TCH55 (lane 3), TCH72 (lane 4), TCH214 (lane 5), TCH217 (lane 6), TCH224 (lane 7), and from $S$. cerevisiae (S.c.). All TCH strains had the API index 2542034. Numbers on the right indicate chromosomal DNA molecular size markers from $S$. cerevisiae.

\section{Electrophoretic karyotypes of C. glabrata}

The 21 isolates of C. glabrata were analysed (Fig. $4 a$ ) using the same conditions as in Fig. 1(a), and chromosomes smaller than $1.2 \mathrm{Mb}$ were analysed using different conditions (Fig. 4b). Their chromosomal DNAs were resolved into $6-12$ bands in the size range $0 \cdot 42-2 \cdot 4 \mathrm{Mb}$ under the conditions of Fig. 4(b). One or two bands larger than $1.4 \mathrm{Mb}$ were very viriable in size among the isolates. The variable bands also hybridized to the rDNA probe (Fig. 2, lanes 9-16) that hybridizes to the variable bands of C. albicans. The band patterns in the size range less than $1.4 \mathrm{Mb}$ were similar among the isolates but at least a few bands were different in each karyotype. Therefore, as with $C$. albicans, we could distinguish $C$. glabrata strains by their karyotypes.

\section{Electrophoretic karyotypes of the other yeasts}

The isolates of C. krusei, C. parapsilosis, $S$. cerevisiae, $R$. rubra and $C r$. uniguttulatus were also analysed by PFGE (data not shown). The isolates of C. krusei, C. parapsilosis and $S$. cerevisiae gave karyotypes similar to those previously reported for these species (Suzuki et al., 1988). DNA bands were not detected in $R$. rubra and $C r$. uniguttulatus, presumably because the DNA preparation method we used was not suitable for these species.

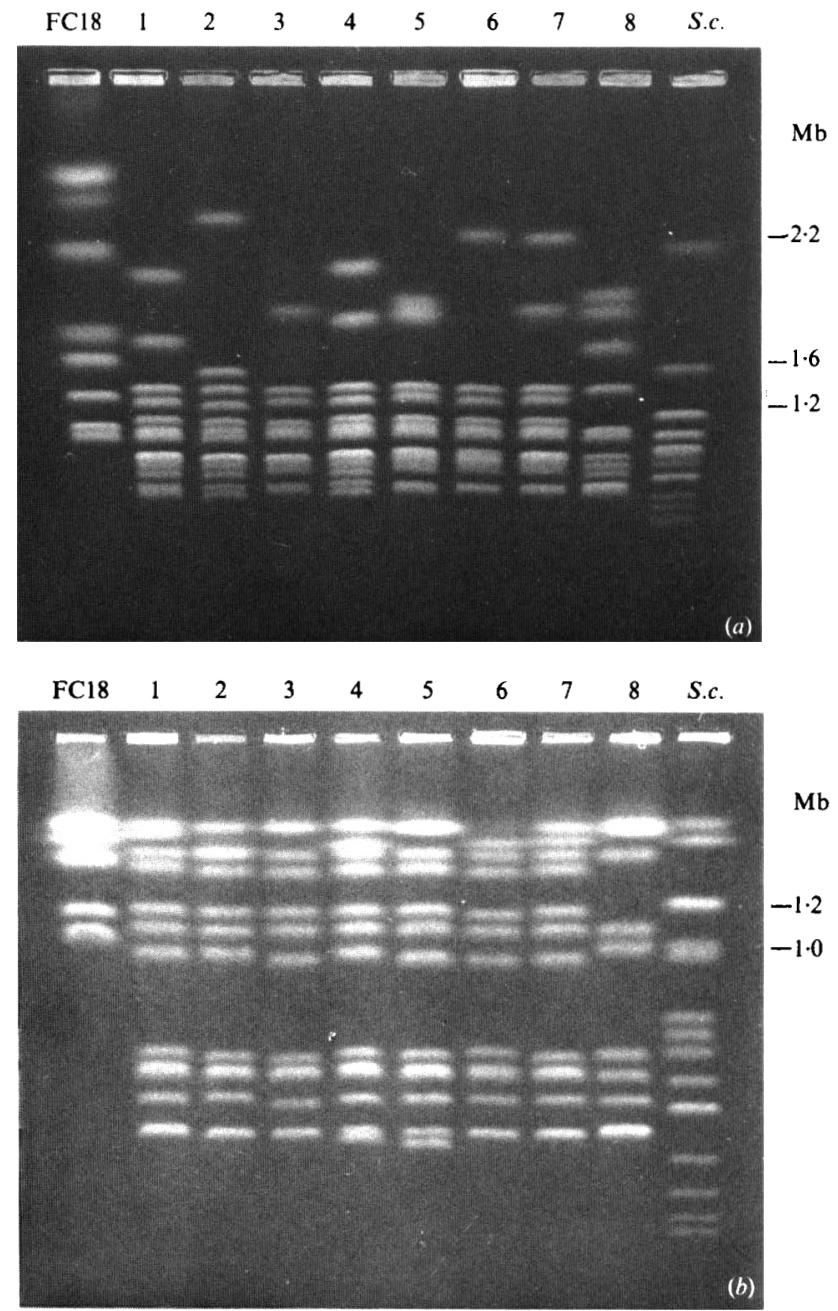

Fig. 4. Separation of chromosomal DNAs of C. glabrata. PFGE was run under conditions of Fig. $1(a)$ and $60 \mathrm{~s}$ for $15 \mathrm{~h}$ at $200 \mathrm{~V}$, then $90 \mathrm{~s}$ for $8 \mathrm{~h}$ at 200 V. (a) was run using the Pulsaphor system (Pharmacia-LKB) and $(b)$ using the CHEF-DRII system (Bio-Rad). The samples were prepared from C. glabrata strains TIMM1063 (lane 1), TCH1 (lane 2), TCH26 (lane 3), TCH58 (lane 4), TCH157 (lane 5), TCH198 (lane 6), TCH 201 (lane 7), TCH220 (lane 8), C. albicans (FC18) and S. cerevisiae (S.c.). Numbers on the right margin indicate chromosomal DNA molecular size markers from $S$. cerevisiae.

Electrophoretic karyotypes of recurrently isolated yeasts.

The karyotypes of isolates which were recovered from seven individual patients (P1-P7) after intervals of 1-6 months were compared (Fig. 5). The patients were treated with clotrimazole (Empecid tablets and cream, Bayer) after the first isolation. C. albicans and C. glabrata were isolated from five and two patients, respectively. The same species were isolated both times. The karyotypes of both isolates from each patient were almost identical except for 1 or 2 bands. The non- 

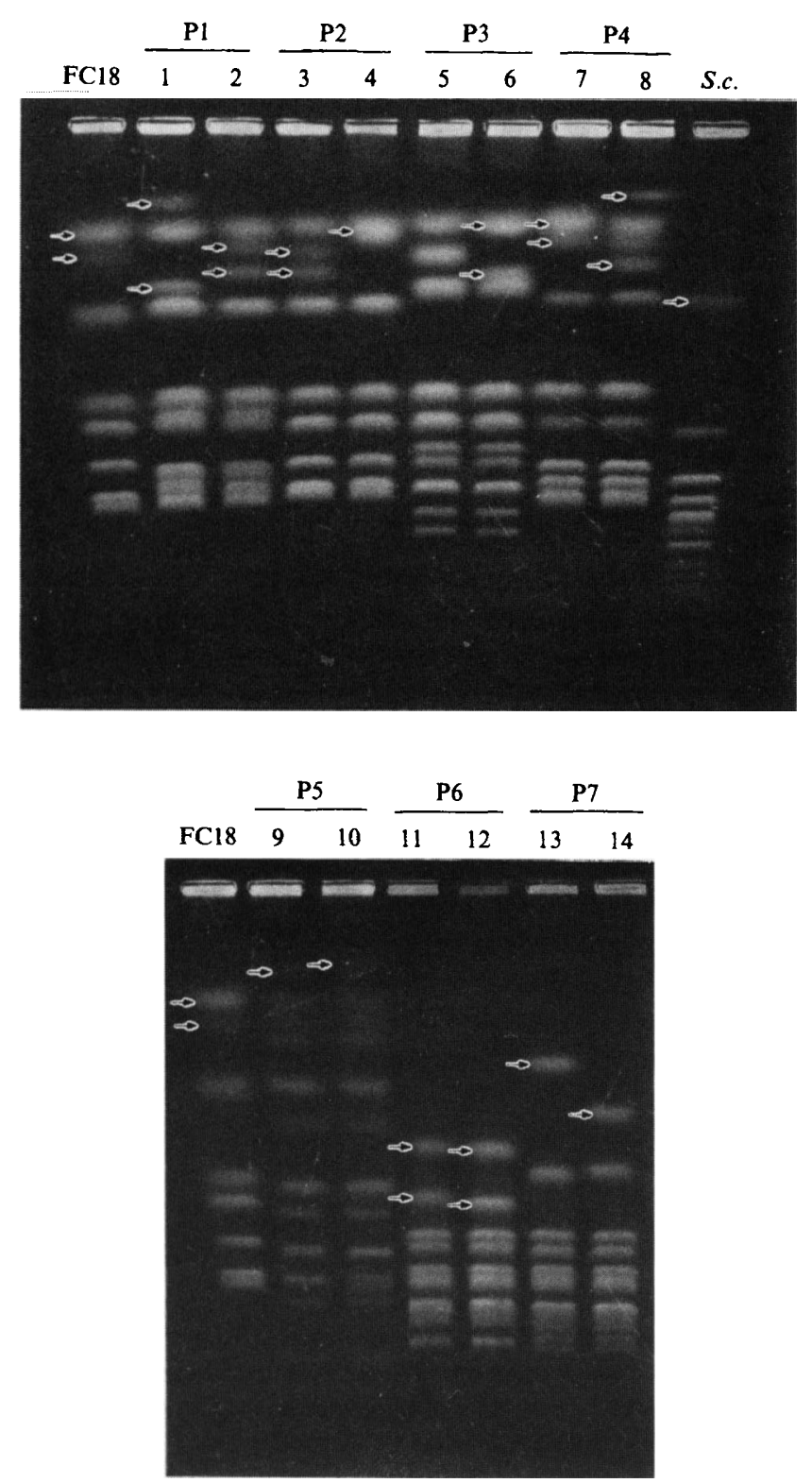

Fig. 5. Comparison of the karyotypes between recurrently isolated yeasts. PFGE was run under the conditions of Fig. 1(a). The sample was prepared from C. albicans strains FC18, TCH32 (lane 1), TCH195 (lane 2), TCH48 (lane 3), TCH215 (lane 4), TCH117 (lane 5), TCH219 (lane 6), TCH136 (lane 7), TCH212 (lane 8), TCH23 (lane 9), TCH217 (lane 10), from C. glabrata strains TCH43 (lane 11), TCH92 (lane 12), TCH58 (lane 13), TCH100 (lane 14), and from S. cerevisiae (S.c.). Arrows at the left of each lane indicate the bands which hybridized to rDNA. TCH117 (lane 5) was not tested for hybridization. The TCH strains were isolated from seven patients (P1-P7). The interval between isolations was 5 months for P1, 5 months for P2, 2 months for P3, 2 months for P4, 6 months for P5, 2 months for P6 and 1 month for P7.

identical bands of the C. albicans isolates were confirmed as variable bands by the rDNA probe (Fig. 5) (see Discussion). In P5, the $1.12 \mathrm{Mb}$ band (the third band from the bottom; Fig. 5, lane 9) detected in the first isolate appeared to be split into two bands in the second isolate. They did not hybridize to the rDNA probe. In the other $C$. glabrata isolates, the non-identical bands larger than $1.4 \mathrm{Mb}$ hybridized to the rDNA probe and so corresponded to the variable bands.

\section{Discussion}

We could distinguish only five types among the isolates of $C$. albicans and only one type of $C$. glabrata by a combination of API index profiles and serotypes. Within the five types of $C$. albicans, the isolates might be further distinguishable by chlamydospore formation, colony morphology, germ tube formation and extracellular proteinase activity. However, it is difficult to get consistent results because these phenotypes are very dependent on physiological conditions and are difficult to judge objectively. In contrast, the karyotypes of the $C$. albicans and $C$. glabrata isolates shared no identical patterns, and thus individual isolates of either species could be distinguished by PFGE karyotypes on well separated gels even without taking into account the variable bands (see below). Though the resolution was less than that shown here and the variable bands were not observed, similar studies have been carried out previously in these species and 17 isolates of $C$. albicans and 33 isolates of C. glabrata were found to give 14 and 22 karyotype patterns respectively (Kaufmann \& Merz, 1989; Merz et al., 1988). From the present study and from our previous study (Iwaguchi et al., 1990), the variability of the karyotypes appears continuous and it is very difficult to classify the karyotype patterns.

In C. albicans, chromosomal DNA band patterns of the isolates described here were similar to that of the standard strain FC18 and the inter-isolate variations were smaller than those of our laboratory strains analysed previously (Iwaguchi et al., 1990). The fresh isolates of this study were recovered from one hospital and from vaginas only. This very limited collection may explain the homogeneous genomic background. However, the possibility that the chromosomal DNAs of our laboratory strains have rearranged during maintenance is also quite possible.

We found a subgroup of seven isolates of $C$. albicans with a consistent API index (2542034) and karyotype. The karyotype was rather similar to that of type I C. stellatoidea, a subspecies of $C$. albicans (KwongChung et al., 1989; Rikkerink et al., 1990; Wickes et al., 1991), but sucrose assimilation was positive (API test) in our isolates, suggesting that our isolates are not $C$. stellatoidea. Furthermore, proteinase activity in the culture medium of five of these seven isolates was about 2-fold higher than the highest activity of the other C. albicans 
isolates (data not shown). Chlamydospores were not observed in any of these seven strains grown on cornmeal/Tween 80 plates and their serotype was classified as B. In spite of their remarkable features, the clinical symptoms they caused could not be distinguished from those caused by the other $C$. albicans isolates. The karyotype differences between these isolates is inferred to be generated by an extensive genomic reorganization (Iwaguchi et al., 1990). Their phenotypic changes, such as high proteinase activity, may be advantageous for survival, and might contribute to their proliferation in the vagina. We aim to investigate the karyotypes of isolates from different sites in the body. It might also be interesting to examine whether this type of strain is found only in the geographical area around Chita, Japan.

Distinct electrophoretic karyotypes were not associated with isolates with the API index 2144174, 6172114 and 2576174, although an atypical karyotype was correlated with an API index of 2542034 as described above. This means that the API index does not always correlate with karyotype. Similarly, no general correlation was found between the karyotype and clinical symptoms, activity of extracellular proteinase, colony morphology or serotypes in this or in our previous study (Iwaguchi et al., 1990). Phenotypes and karyotypes need not be directly correlated but karyotype changes can provide genetic variation and may thereby lead to phenotypic changes. In this regard, association of karyotype changes and colony morphology has been studied extensively (Rustchenko-Bulgac et al., 1990; Suzuki et al., 1989).

In $C$. glabrata, the chromosome number has not been determined although an estimation was reported on the basis of an electrophoretic karyotype analysis (Kaufman \& Merz, 1989). Hybridization studies are necessary to clarify the chromosome organization of this species. At this time, probe DNAs specific for $C$. glabrata are not available but probes of a tubulin gene, an actin gene and an rDNA gene hybridized are all known to the DNA of C. glabrata (Mason et al., 1987; Iwaguchi et al., 1990; Magee et al., 1988). In the present study, one or two bands larger than $1.4 \mathrm{Mb}$ were very variable among the isolates of C. glabrata and these variable bands hybridized to the rDNA probe. The rDNA gene sequence is usually highly repeated in a single region and DNA rearrangements occur at high frequency in this region (Szostak \& Wu, 1980); such rearrangements may be responsible for the change in chromosome size for both C. albicans and C. glabrata.

The karyotypes of isolates of $C$. albicans and $C$. glabrata taken from the same individuals after intervals of 1-6 months were almost identical except for one or two bands for both $C$. albicans and $C$. glabrata. In all cases except that of one band of the isolate from P5 (Fig. 5), these non-identical bands were shown to be the variable bands defined as those hybridizing to the rDNA probe. Thus, these non-identical bands might be derived from high-frequency rearrangement of the chromosome during the time between isolations. We have found that the clonal variation in size of the variable bands occurs at a frequency as high as about $10 \%$ of the progeny clones (unpublished). Both isolates from the same person are presumed to be the same strain because their karyotypes were almost always exactly the same, except for the nonidentical bands. We conclude that the strains from individual patients do not change, at least over short periods. A similar conclusion in recurrent vaginal infection by $C$. albicans has been reached using Southern blot hybridization patterns with a mid-repeat sequence (Soll et al., 1989).

In conclusion, all strains from different individuals could be distinguished one from another by their karyotypes, which were well separated under suitable conditions, and karyotypes seem not to change, at least over short periods, except for the variable chromosome bands. These results suggest that karyotyping permits very precise identification of different strains providing the variable bands are ignored. We think this will be a powerful tool in epidemiological studies for determining the origins of various Candida strains.

We thank Professor P. T. Magee (University of Minnesota) for critically reading the manuscript and Professor $S$. Awaya (Department of Ophthalmology, Nagoya University) for encouragement and support.

\section{References}

Buesching, W. J., KureK, K. \& RoberTS, G. D. (1979). Evaluation of the modified API 20C system for identification of clinically important yeasts. Journal of Clinical Microbiology 9, 565-569.

FoX, B. C., Mobley, H. L.T. \& WADE, J. C. (1989). The use of a DNA probe for epidemiological studies of candidiasis in immunocompromised hosts. Journal of Infectious Diseases 159, 488-494.

IwaGuchi, S.-I., Homma, M. \& TANAKA, K. (1990). Variation in the electrophoretic karyotype analysed by the assignment of DNA probes in Candida albicans. Journal of General Microbiology 136, 2433-2442.

Kaufmann, C. S. \& Merz, W. G. (1989). Electrophoretic karyotypes of Torulopsis glabrata. Journal of Clinical Microbiology 27, 21652168.

KwoNG-Chung, K. J., Riggsby, W. S., UphofF, R. A., Hicks, J. B. \& WhELAN, W. L. (1989). Genetic differences between Type I and Type II Candida stellatoidea. Infection and Immunity 57, 527-532.

Lasker, B. A., Carle, G. F., Kobayashi, G. S. \& Medoff, G. (1989). Comparison of the separation of Candida albicans chromosome-sized DNA by pulsed-field gel electrophoresis techniques. Nucleic Acids Research 17, 3783-3793.

Magee, B. B., Koltin, Y., Gorman, J. A. \& Magee, P. T. (1988). Assignment of cloned genes to the seven electrophoretically separated Candida albicans chromosomes. Molecular and Cellular Biology 8, 4721-4726.

Mahrous, M., Lott, T. J., Meyer, S. A., Sawant, A. D. \& Ahearn, D. G. (1990). Electrophoretic karyotyping of typical and atypical Candida albicans. Journal of Clinical Microbiology 28, 876-881. 
Mason, M. M., Lasker, B. A. \& Riggsby, W. S. (1987). Molecular probe for identification of medically important Candida species and Torulopsis glabrata. Journal of Clinical Microbiology 25, 563-566.

Merz, W. G. (1990). Candida albicans strain delineation. Clinical Microbiology Reviews 3, 321-334.

Merz, W. G., Connelly, C. \& Hieter, P. (1988). Variation of electrophoretic karyotypes among clinical isolates of Candida albicans. Journal of Clinical Microbiology 26, 842-845.

Monod, M., Porchet, S., Baudraz-Rosselet, F. B. \& Frenk, E. (1990). The identification of pathogenic yeast strains by electrophoretic analysis of their chromosomes. Journal of Medical Microbiology 32, 123-129.

MORTIMER, R. K. \& SCHILD, D. (1985). Genetic map of Saccharomyces cerevisiae, edition 9. Microbiological Reviews 49, 181-212.

Rikkerink, E. H., Magee, B. B. \& Magee, P. T. (1990). Genomic structure of Candida stellatoidea: extra chromosomes and gene duplication. Infection and Immunity 58, 949-954.

Rustchenko-Bulgac, E. P., Sherman, F. \& Hicks, J. B. (1990). Chromosomal rearrangements associated with morphological mutants provide a means for genetic variation of Candida albicans. Journal of Bacteriology 172, 1276-1283.

SCherer, S. \& MAGEe, P. T. (1990). Genetics of Candida albicans. Microbiological Reviews 54, 226-241.

SCHERER, S. \& STEVENS, D. A. (1987). Application of DNA typing methods to epidemiology and taxonomy of Candida species. Journal of Clinical Microbiology 25, 675-679.

SCHWARTZ, D. C. \& CANTOR, C. R. (1984). Separation of yeast chromosome-sized DNAs by pulse field gradient gel electrophoresis. Cell 37, 67-75.
Shinoda, T., Kaufman, L. \& Padhye, A. A. (1981). Comparative evaluation of the Iatron serological candida check kit and the API 20 C kit for identification of medically important Candida species. Journal of Clinical Microbiology 13, 513-578.

Soll, D. R., Galask, R., Isley, S., Rao, T. V. G., Stone, D., Hicks, J., Schmid, J., MAC, K. \& Hanna, C. (1989). Switching of Candida albicans during successive episodes of recurrent vaginitis. Journal of Clinical Microbiology 27, 681-690.

Sugihara, K., OHIShI, K. \& Toh-E, A. (1986). Ribosomal DNA plasmid isolated from Zygosaccharomyces bailii and its use for constructing yeast vectors effective for intergeneric gene transfer. Agricultural and Biological Chemistry 50, 1503-1512.

Suzuki, T., Kobayashi, I., Mizuguchi, I., Banno, I. \& Tanaka, K. (1988). Electrophoretic karyotypes in medically important Candida species. Journal of General and Applied Microbiology 34, 409-416.

Suzuki, T., Kobayashi, I., Kanbe, T. \& Tanaka, K. (1989). High frequency variation of colony morphology and chromosome reorganization in the pathogenic yeast Candida albicans. Journal of General Microbiology 135, 425-434.

Szostak, J. W. \& WU, R. (1980). Unequal crossing over in the ribosomal DNA of Saccharomyces cerevisiae. Nature, London 284, 426-430.

VollRath, D. \& Davis, R. W. (1987). Resolution of DNA molecules greater than 5 megabases by contour-clamped homogenous electric fields. Nucleic Acids Research 15, 7865-7876.

Wickes, B. L., Golin, J. E. \& Kwon-Chung, K. J. (1991). Chromosomal rearrangement in Candida stellatoidea results in a positive effect on phenotype. Infection and Immunity 59, 1762-1771. 